International Journal of Quantum Information

(C) World Scientific Publishing Company

\title{
POVM construction: a simple recipe with applications to symmetric states
}

\author{
SWARNAMALA SIRSI
}

Yuvaraja's College, University of Mysore, Mysuru,

India.

swarnamalasirsi@ycm.uni-mysore.ac.in

KARTHIK BHARATH

University of Nottingham, Nottingham,

U.K.

karthik.bharath@nottingham.ac.uk

S. P. SHILPASHREE

K. S. School of Engineering and Management,

Bangalore,

India.

shilpashreesp@gmail.com

H. S. SMITHA RAO

Yuvaraja's College, University of Mysore,

Mysuru,

India.

cosmosmitha.rao@gmail.com

Received Day Month Year

Revised Day Month Year

\begin{abstract}
We propose a simple method for constructing POVMs using any set of matrices which form an orthonormal basis for the space of complex matrices. Considering the orthonormal set of irreducible spherical tensors, we examine the properties of the construction on the $n+1$-dimensional subspace of the $2^{n}$-dimensional Hilbert space of $n$ qubits comprising the permutationally symmetric states. Using the notion of vectorization, the constructed POVMs are interpretable as projection operators in a higher-dimensional space. We then describe a method to physically realize the constructed POVMs for symmetric states using the Clebsch-Gordan decomposition of the tensor product of irreducible representations of the rotation group. We illustrate the proposed construction on a spin-1 system, and show that it is possible to generate entangled states from separable ones.
\end{abstract}

Keywords: permutationally symmetric states; projection operators; POVM. 


\section{Introduction}

Positive Operator-Valued Measures (POVMs) are used frequently in quantum information processing tasks including quantum filtering, 12 realization of quantum communication protocols, ${ }^{3 / 6}$ entanglement verification,${ }^{7}$ Remote State Preparation $(\mathrm{RSP})^{\sqrt[8]{8}}$ and conclusive teleportation $\sqrt[9]{10}$ Their construction and physical realization are of particular interest in quantum information theory ${ }^{11}[13$ and in the proof of quantum de Finetti theorem, $\frac{14}{14}$ with emphasis on optimal measurements through Symmetric Informationally-Complete (SIC) POVMs $\frac{15] 16}{16}$ Their importance lies in the fact that they can be used to achieve certain tasks which are outside the scope of projective measurements; for example, a set of non-orthogonal states cannot be distinguished using projective measurements, but can be discriminated unambiguously using POVMs $\frac{17] 18}{18}$ A general construction mechanism for POVMs is therefore of considerable importance.

On the other hand, POVMs which assist in obtaining specific post-measurement states, and possess a simple physical realization mechanism, can be harnessed for various tasks in quantum information processing. When the density matrix representing a state is viewed as an element of a linear vector space of complex matrices, classification of the states based on the choice of orthonormal basis matrices becomes possible. For example, under the representation of a density matrix in the spherical tensor basis proposed by Fano, $\frac{19}{19}$ it is possible to classify states based on the rank of the tensors required to construct the corresponding density matrices: density matrices with only spherical tensors of rank 1 correspond to states which are vector-polarized and are known as 'oriented systems' in literature $\frac{20}{20}$ rank 2-tensor polarised states correspond to density matrices which have a representation with only rank 2 -spherical tensors. An aligned spin- 1 system, which can be generated in the laboratory by exposing spin-1 nuclei like deuterons with non-zero quadrupole moment to external electric quadrupole fields with non-zero asymmetry parameter, is one such example..$^{21}$ As a consequence, given a density matrix for an arbitrary state, such a classification leads to interest in constructing POVMs tailored to result in a post-measurement state polarized by tensors of a specific rank.

In this Letter we propose a simple but general mechanism for POVM construction based on any orthonormal set of basis matrices on the vector space of complex matrices. The construction leads to a novel interpretation of the POVMs as orthogonal projectors in a space of higher dimension. The interpretation of the action of the POVM becomes transparent using the notion of vectorization, with the post-measurement state suitably viewed as one being projected onto the subspace spanned by chosen bases. This leads to interpreting the post-measurement state as a pure state in the higher-dimensional space. For the case of permutationally symmetric states, we additionally provide an efficient physical realization mechanism for the POVMs based on the Clebsch-Gordan decomposition of tensor products into irreducible representations of $S U(2)^{\otimes n}$. Such a procedure is similar in spirit to the construction of projective measurements from POVMs through 
Neumark's theorem $\frac{221}{22}$ but operationally very different. The POVMs dilated to the $2^{n}$-dimensional Hilbert space are not projective measurements, and generally not repeatable. Nevertheless, repeatability can be viewed in the sense that the dilated POVM always projects onto the subspace of symmetric states. We demonstrate the physical implementation of the POVMs in a 4-dimensional 2-qubit Hilbert space, and additionally, demonstrate a mechanism to generate entangled states from initial separable states. For $n$ qubits residing in the $2^{n}$-dimensional Hilbert space, the $n+1$-dimensional subspace of permutationally symmetric states corresponds to an experimentally developed and rich class $\frac{23}{24}$ in quantum information processing; the class includes Bell states, Greenberger-Horne-Zeilinger (GHZ) states, W states, and $n$-qubit Dicke states, and POVMs on this class are crucial in characterization of nonlocality $\sqrt{25}$ and multipartite entanglement $\sqrt{26}$ Given their utility in such tasks, construction of POVMs whose action always lead to symmetric post-measurement states can be particularly useful.

\section{General POVM construction}

Let $\mathcal{M}_{N}$ denote the vector space of $N \times N$ matrices of dimension $N^{2}$ over the field of complex numbers $\mathbb{C}$. Define a map $\mathcal{V}: \mathcal{M}_{N} \rightarrow \mathbb{C}^{N^{2}}, \mathcal{V}(X)=\left(x_{1}, x_{2}, \cdots, x_{N}\right)^{T}$, where $x_{i}$ is the $i$ th row of $X$ containing $N$ elements, and $v$ denotes the transpose of a vector $v$; the map transforms an $N \times N$ matrix into a column vector of $N^{2}$ elements by stacking rows into a column, providing an equivalent representation of elements of $\mathcal{M}_{N}$. The map $\mathcal{V}$ is a linear map which expresses an isomorphism $\mathcal{M}_{N}:=\mathcal{C}^{N} \otimes \mathcal{C}^{N} \cong \mathbb{C}^{N^{2}}$, and represents a unitary transformation from the space of $N \times N$ matrices with complex entries with the Hilbert-Schmidt inner product to $\mathbb{C}^{N^{2}}$ equipped with usual inner product (sesquilinear form), as evidenced by the equality $\operatorname{Tr}\left(X^{\dagger} Y\right)=\left\langle\mathcal{V}(X)^{*}, \mathcal{V}(Y)\right\rangle$. In order to see that $\mathcal{V}$ is linear map, let $\mathbf{C}_{i}$ be an $N^{2} \times N$ block matrix containing the identity $\mathbb{I}_{N}$ in the $i$ th position and $\mathbf{0}$ matrices everywhere else. That is, $\mathbf{C}_{i}$ consists of $N$ block matrices of size $N \times N$ stacked column-wise containing the identity matrix $\mathbb{I}_{N}$ in the $i$ th position. Suppose $\left\{e_{i}, i=1, \ldots, N\right\}$ is the canonical basis in $\mathbb{C}^{N}$, then it can be verified that $\mathcal{V}(A)=\sum_{i=1}^{N} \mathbf{C}_{i} A e_{i} \quad A \in \mathcal{M}_{N}$.

The inverse map $\mathcal{V}^{-1}: \mathbb{C}^{N^{2}} \rightarrow \mathcal{M}_{N}$ is then the unique map which constructs an $N \times N$ complex matrix by taking the first $N$ elements of $\mathcal{V}(X)$ and setting that as the first row of $X$, and so on. Since $\mathcal{M}_{N}$ is a vector space over $\mathbb{C}$, we can choose the elementary matrix set $\left\{E_{i j}: i, j=1, \ldots, N\right\}$ of $N \times N$ matrices with 1 in the $(i, j)$ th position and 0 everywhere else as an orthonormal basis; indeed, $\left(E_{i j}^{\dagger} E_{k l}\right)=\delta_{i k} \delta_{j l}$ and $\left\{E_{i j}\right\}$ forms an orthonormal set of basis matrices for $\mathcal{M}_{N}$. Any element of $\mathcal{M}_{N}$ can be expressed as a linear combination, with complex coefficients, of the $E_{i j} \mathrm{~s}$. It is easy to verify that $E_{i j} E_{i j}^{\dagger}$ is Hermitian, non-negative for each $i, j$, and they satisfy the partition of unity condition $\sum_{i, j} E_{i j} E_{i j}^{\dagger}=N \mathbb{I}_{N}$.

Let us denote by $\left\{T_{i j}: i, j=1, \ldots, N\right\}$ another set of orthonormal basis matrices for $\mathcal{M}_{N}$. If $U$ is an $N^{2} \times N^{2}$ unitary matrix with $U U^{\dagger}=\mathbb{I}_{N^{2}}$, then it is possible to 
obtain a new orthonormal basis $\left\{\mathcal{V} T_{i j}\right\}$, where $\left\{\mathcal{V} T_{i j}=U \mathcal{V}\left(E_{i j}\right): i, j=1, \ldots, N\right\}$. Explicitly, $\mathcal{V} T_{i j}=U \mathcal{V}\left(E_{i j}\right)=\left(u_{i j}^{(1)}, u_{i j}^{(2)}, \cdots, u_{i j}^{(N)}\right)^{T}$, where the $N$-dimensional $u_{i j}^{(k)}$ is the $k$ th sub-vector of the $N^{2}$-dimensional complex vector $\mathcal{V} T_{i j}$. The inverse mapping $\mathcal{V}^{-1}$, along with unitary matrix $U$, provides us with way to obtain the $N \times N$ orthonormal basis matrices $\left\{T_{i j}\right\}$ from $\left\{E_{i j}\right\}$ as $T_{i j}=\mathcal{V}^{-1}\left(U \mathcal{V}\left(E_{i j}\right)\right)=$ $\left(u_{i j}^{(1)}, u_{i j}^{(2)}, \cdots, u_{i j}^{(N)}\right)^{T}$. We have thus shown how any orthonormal basis matrices for $\mathcal{M}_{N}$ can be constructed from the elementary basis $E_{i j}$. Note that for any complex matrix $A$, not necessarily square, $A A^{\dagger}$ is always non-negative and Hermitian. From the partition of unity condition and the fact that $U U^{\dagger}=\mathbb{I}_{N^{2}}$, it is now straightforward to verify that $\sum_{i, j} T_{i j} T_{i j}^{\dagger}=\alpha \mathbb{I}_{N}$, where $\alpha$ is a constant not depending on $i$ and $j$.

Theorem 1. Let $\left\{T_{j}: j=1, \ldots, N^{2}\right\}$ be an orthonormal set of basis matrices for $\mathcal{M}_{N}$. Then the set $\left\{\alpha T_{j} T_{j}^{\dagger}\right\}$ satisfies the conditions of a POVM, where $\alpha$ is a constant not depending on $j$.

The $T_{i}^{\dagger}$ arise from the linear map $A \mapsto \mathcal{V}^{-1} U \mathcal{V}(A)$ used in Theorem 1 and this map is not positive (and hence not completely positive). However, as with the Kraus operators, the $T_{i}^{\dagger}$ that enable the POVM construct are unitary equivalent, and hence in this sense play the role of Kraus operators. 29

\section{POVM Construction and physical realization for symmetric states}

Here we implement the POVM construction mechanism on permutationally symmetric states.

Permutationally symmetric states. - Set of $n$-qubit states that remains unchanged by permutation of individual particles are called symmetric states. Specifically, if $\pi_{k, l}$ is the permutation operator on the set $\{1,2, \ldots, n\}$ that permutes $k$ and $l$ with $k \neq l$ and $k, l \in\{1,2, \ldots, n\}$, and $\rho_{1,2, \ldots, n}^{s}$ is the density matrix for the $n$-qubit symmetric state, $\pi_{k, l} \rho_{1,2, \ldots, n}^{s} \pi_{k, l}^{\dagger}=\rho_{1,2, \ldots, n}^{s}$. A general $n$-qubit symmetric state belongs to the Hilbert space $\mathbb{C}^{2 \otimes n}$ and its density matrix is of dimension $2^{n} \times 2^{n}$ whereas the $n$-qubit symmetric state resides in a Hilbert space of dimension $n+1$. This symmetric subspace can be identified with a $2 j+1$-dimensional Hilbert space, which is the carrier space for the angular momentum operator $\mathbf{J}=\left(J_{x}, J_{y}, J_{z}\right)$. Thus the $n$-qubit symmetric state has a one-to-one correspondence with a spin- $j$ state with $j=n / 2$.

Spherical tensor representation of the density matrix.- Henceforth, suppressing notation, $\rho$ represents an $n$-qubit symmetric state. The density matrix for a general 
$n$-qubit symmetric state or a spin- $j$ system can be represented as

$$
\rho(\mathbf{J}):=\rho:=\frac{1}{(2 j+1)} \sum_{k=0}^{2 j} \sum_{q=-k}^{k} t_{q}^{k} \tau_{q}^{k^{\dagger}},
$$

where $j=n / 2$ and $\tau_{q}^{k}$ are irreducible tensor operators of rank $k$ in the $N=n+1$ dimensional spin space with projection $q$ along the axis of quantization in the real 3-dimensional space, where $\tau_{0}^{0}=\mathbb{I}_{N}$, the $N \times N$ identity operator; this representation is due to Fano ${ }^{19}$ Here and elsewhere, orthogonality in the matrix space is always defined with respect to the Hilbert-Schmidt inner product. The $\tau_{q}^{k}$ satisfy the relations, $\left(\tau_{q}^{k^{\dagger}} \tau_{q^{\prime}}^{k^{\prime}}\right)=(2 j+1) \delta_{k k^{\prime}} \delta_{q q^{\prime}}, \quad \tau_{q}^{k^{\dagger}}=(-1)^{q} \tau_{-q}^{k}$, where the normalization has been chosen so as to be in agreement with Madison convention. ${ }^{30}$ The Fano statistical tensors or the spherical tensor parameters $t_{q}^{k}$ parametrize the density matrix $\rho$ as expectation values of $\tau_{q}^{k}:\left(\rho \tau_{q}^{k}\right)=t_{q}^{k}$. Because of hermiticity of $\rho, t_{q}^{k^{*}}=(-1)^{q} t_{-q}^{k}$. In other words, the $N^{2}-1$ spherical tensor operators $\tau_{q}^{k}$, in conjunction with the identity operator, form an orthonormal basis for the vector space of $N \times N$ complex matrices over $\mathbb{C}$ that act on the $N$-dimensional spin space. In contrast to some orthonormal basis matrices, like Gell-Mann matrices, their importance lies in the fact they can be constructed as symmetrized products of the angular momentum operators $\mathbf{J}$ following the well-known Weyl construction ${ }^{31}$ as, $\tau_{q}^{k}(\mathbf{J})=\mathcal{N}_{k j}(\mathbf{J} \cdot \vec{\nabla})^{k} r^{k} Y_{q}^{k}(\hat{r})$, where $\mathcal{N}_{k j}$ are the normalization factors and $Y_{q}^{k}(\hat{r})$ are the spherical harmonics. For $j=1$, from the expressions for $\tau_{q}^{k} \mathrm{~s}$ in terms of $J_{x}, J_{y}, J_{z}$, it is clear that $\tau^{1} \mathrm{~s}$ are anti-symmetric whereas $\tau^{2} \mathrm{~s}$ are symmetric under the permutation of indices $x, y$ and $z$. The $\tau_{q}^{k}$ s possess simple transformation properties under coordinate rotations of the 3 -dimensional space: for a rotation $R(\alpha, \beta, \gamma)$, where $\alpha, \beta$ and $\gamma$ are Euler angles, the parameters in the rotated coordinates, $\left(t_{q}^{k}\right)^{R}$, are related to the ones in the initial coordinates as $\left(t_{q}^{k}\right)^{R}=\sum_{q^{\prime}} D_{q^{\prime} q}^{k}(\alpha, \beta, \gamma) t_{q}^{k}$, where $D_{q^{\prime} q}^{k}(\alpha, \beta, \gamma)$ are the Wigner rotation matrices; thus the rank of the tensor is preserved under rotations. The matrix elements of the tensor operators, in the angular momentum basis, are given by $\left\langle j m^{\prime}\left|\tau_{q}^{k}(\mathbf{J})\right| j m\right\rangle=[k] C\left(j k j ; m q m^{\prime}\right)$, where $C\left(j k j ; m q m^{\prime}\right)$ are the Clebsch-Gordan coefficients and $[k]=\sqrt{2 k+1}$. The tensor operators are traceless but not Hermitian, and cannot in general be identified with generators of $S U(N)$.

POVM construction. - The subspace of permutationally symmetric states is spanned by the eigen states $|j m\rangle$ of angular momentum operators $\mathbf{J}^{2}$ and $J_{z}$, where $j=n / 2$ and $m=-j, \ldots,+j$. Employing Theorem 1 in conjunction with the orthonormal set of spherical tensors from (1) which forms a basis for the space of $N \times N$ complex matrices, we can construct POVMs for a symmetric subspace of dimension $(2 j+1)$, as

$$
E_{q}^{k}=\frac{\tau_{q}^{k} \tau_{q}^{k^{\dagger}}}{\mathcal{N}^{2}}, \quad k=0, \ldots, 2 j ; q=-k, \cdots, k,
$$


where $\mathcal{N}$ is a constant not depending on $k$ and $q$. Interestingly, using symmetry properties of Clebsch-Gordan coefficients, it can be verified that the set $\left\{E_{q}^{k}\right\}$ contains only diagonal matrices as follows. Evidently, $\left(\tau_{q}^{k} \tau_{q}^{k^{\dagger}}\right)^{\dagger}=\left(\tau_{q}^{k^{\dagger}}\right)^{\dagger} \tau_{q}^{k^{\dagger}}=\tau_{q}^{k} \tau_{q}^{k^{\dagger}}$, $E_{q}^{k}$ is Hermitian for all $k$ and $q$. Clearly, $E_{q}^{k}$ is non-negative from elementary properties of complex matrices. The only condition which requires checking is resolution of the identity operator; this will be done using the $|j m\rangle$ basis with $k^{\prime}$ and $q^{\prime}$ indexing the elements of the POVMs. Consider the matrix elements of $E_{q}^{k}$ in the angular momentum basis, with indices in the summation indexing the elements of the POVMs:

$$
\begin{aligned}
\left\langle j m\left|\sum_{k^{\prime} q^{\prime}} E_{q^{\prime}}^{k^{\prime}}\right| j m^{\prime}\right\rangle= & \left\langle j m\left|\sum_{k^{\prime} q^{\prime}} \tau_{q^{\prime}}^{k^{\prime}} \tau_{q^{\prime}}^{k^{\prime \dagger}}\right| j m^{\prime}\right\rangle \\
& =\sum_{m^{\prime \prime} k^{\prime} q^{\prime}}(-1)^{q^{\prime}}\left\langle j m\left|\tau_{q^{\prime}}^{k^{\prime}}\right| j m^{\prime \prime}\right\rangle\left\langle j m^{\prime \prime}\left|\tau_{-q^{\prime}}^{k^{\prime}}\right| j m^{\prime}\right\rangle \\
& =\sum_{m^{\prime \prime} k^{\prime} q^{\prime}}(-1)^{q^{\prime}}\left[k^{\prime}\right]^{2} C\left(j k^{\prime} j ; m^{\prime \prime} q^{\prime} m\right) C\left(j k^{\prime} j ; m^{\prime}-q^{\prime} m^{\prime \prime}\right) .
\end{aligned}
$$

Using the symmetry properties of Clebsch-Gordan coefficients, we obtain $\left\langle j m\left|\sum_{k^{\prime} q^{\prime}} E_{q^{\prime}}^{k^{\prime}}\right| j m^{\prime}\right\rangle=\sum_{k^{\prime}}\left[k^{\prime}\right]^{2} \delta_{m m^{\prime}} ;$ and thus, $\frac{1}{\mathcal{N}^{2}}\left\langle j m\left|\sum_{k^{\prime} q^{\prime}} E_{q^{\prime}}^{k^{\prime}}\right| j m^{\prime}\right\rangle=\delta_{m m^{\prime}}$ with $\mathcal{N}^{2}=\sum_{k^{\prime}}\left[k^{\prime}\right]^{2}$.

Post-measurement state and its interpretation.- Using the constructed POVM, a measurement on an initial state $\rho^{i}$ results in a final state, described by the density operator

$$
\rho_{k q}^{f}=\frac{E_{q}^{k} \rho^{i} E_{q}^{k}}{\operatorname{Tr}\left(E_{q}^{k} \rho^{i} E_{q}^{k}\right)} .
$$

It is evident that $\rho_{k, q}^{f}$ is a valid density matrix. However its interpretation as an update rule following measurement is not clear. But this issue can be made addressed through the vectorization map $\mathcal{V}$ discussed earlier. Using the map we transform each of the the $N \times N$ matrices in equation (3) to $N^{2} \times N^{2}$ dimension matrices. For example, $\mathcal{E}_{q}^{k}:=\left|\tau_{q}^{k}\right\rangle\left\langle\tau_{q}^{k}\right|$ can be viewed as an $N^{2} \times N^{2}$ matrix where $\left|\tau_{q}^{k}\right\rangle:=\mathcal{V}\left(\tau_{q}^{k}\right)$. In order to transform $\rho^{i}$, consider the matrix $\rho_{v}^{i}:=\left|\mathcal{V}\left(\rho^{i}\right)\right\rangle\left\langle\mathcal{V}\left(\rho^{i}\right)\right|$, which can be viewed as the density matrix of a pure state in $N^{2}$-dimensional space. If $U$ diagonalizes $\rho_{v}^{i}$, define the diagonal matrix $D_{\rho_{i}}:=U^{\dagger} \rho_{v}^{i} U$, and let $\left|\Omega^{i}\right\rangle=U \sqrt{D_{\rho_{i}}}$. Using these quantities, the RHS of equation (3), which represents the action of the POVM, can be written as

$$
\frac{\left|\tau_{q}^{k}\right\rangle\left\langle\tau_{q}^{k} \mid \Omega^{i}\right\rangle\left\langle\Omega^{i} \mid \tau_{q}^{k}\right\rangle\left\langle\tau_{q}^{k}\right|}{\operatorname{Tr}\left(\left|\tau_{q}^{k}\right\rangle\left\langle\tau_{q}^{k} \mid \Omega^{i}\right\rangle\left\langle\Omega^{i} \mid \tau_{q}^{k}\right\rangle\left\langle\tau_{q}^{k}\right|\right)}=\left|\tau_{q}^{k}\right\rangle\left\langle\tau_{q}^{k}\right|
$$

Thus, we are left to conclude that in the language of $N^{2} \times N^{2}$ matrices, the resulting state $\rho_{k q}^{f}=\mathcal{E}_{q}^{k}$. In the $N^{2}$-dimensional representation, evidently $\left|\tau_{q}^{k}\right\rangle\left\langle\tau_{q}^{k}\right|$ 
are the projection operators, which project the initial state $\left|\Omega^{i}\right\rangle$ 'along' the $\left|\tau_{q}^{k}\right\rangle$ direction. Furthermore, using the transformational properties of the spherical tensors described earlier, we note that a POVM constructed out of $\tau_{q}^{k}$ s projects the given initial density matrix to the $(2 k+1)$ dimensional invariant subspace after the measurement.

Physical realization. - Recall that $n$ qubits reside in a $2^{n}$-dimensional Hilbert space of which the symmetric space of dimension $N=n+1$ is a subspace. In this setting, Neumark's theorem ${ }^{22}$ states that any POVM on the $N$-dimensional symmetric subspace can be realized as a projective measurement in the $2^{n}$ dimensional Hilbert space. We adopt an alternative route by seeking recourse to Clebsch-Gordan decomposition of irreducible representations of $S U(2)^{\otimes n}$. From the tensor product representation of $n$-qubit state the Clebsch-Gordan decomposition leads to a direct sum of irreducible representations of which, the one with the largest dimension corresponds to the invariant subspace of symmetric states. In other words, we are able to obtain an orthogonal decomposition of the $2^{n}$-dimensional Hilbert space into invariant subspaces including symmetric and anti-symmetric states. In essence, the decomposition is achieved through the action of a unitary matrix with ClebschGordan coefficients as elements. What is of interest in this context is that the unitary matrix decomposes the computational basis in the $2^{n}$-dimensional Hilbert space to a set of bases, one amongst which is the basis for the $N$-dimensional symmetric subspace. As an indirect consequence, we are provided with a mechanism to dilate a POVM constructed on the symmetric subspace to the $2^{n}$-dimensional Hilbert space. The key difference with Neumark's theorem is that the dilated POVM is not a projective measurement ensuring repeatability of measurements; instead, repeatability is to be viewed in the sense that measurements based on the dilated POVM will always result in a symmetric state.

\section{Spin-1 example}

As an illustration, we now explicitly construct the following POVMs for a spin-1 system $(n=2$ and $N=3)$, where the $E_{q}^{k}$ s are expressed in the basis $|11\rangle,|10\rangle$ and $|1-1\rangle$ :

$$
\begin{gathered}
E_{0}^{0}=\left(\begin{array}{ccc}
\frac{1}{9} & 0 & 0 \\
0 & \frac{1}{9} & 0 \\
0 & 0 & \frac{1}{9}
\end{array}\right), E_{0}^{1}=\left(\begin{array}{ccc}
\frac{1}{6} & 0 & 0 \\
0 & 0 & 0 \\
0 & 0 & \frac{1}{6}
\end{array}\right), E_{0}^{2}=\left(\begin{array}{ccc}
\frac{1}{18} & 0 & 0 \\
0 & \frac{4}{18} & 0 \\
0 & 0 & \frac{1}{18}
\end{array}\right), E_{1}^{1}=E_{1}^{2}=\left(\begin{array}{ccc}
\frac{1}{6} & 0 & 0 \\
0 & \frac{1}{6} & 0 \\
0 & 0 & 0
\end{array}\right), \\
E_{-1}^{1}=E_{-1}^{2}=\left(\begin{array}{ccc}
0 & 0 & 0 \\
0 & \frac{1}{6} & 0 \\
0 & 0 & \frac{1}{6}
\end{array}\right), \quad E_{2}^{2}=\left(\begin{array}{ccc}
\frac{1}{3} & 0 & 0 \\
0 & 0 & 0 \\
0 & 0 & 0
\end{array}\right), \quad E_{-2}^{2}=\left(\begin{array}{lll}
0 & 0 & 0 \\
0 & 0 & 0 \\
0 & 0 & \frac{1}{3}
\end{array}\right) .
\end{gathered}
$$

We can observe the degeneracy regarding the number of distinct POVM elements; we also note that $E_{q}^{k}$ s do not transform like spherical tensor operators. Starting with a POVM in a 3 -dimensional symmetric subspace we can obtain its dilation to 
the 4-dimensional 2-qubit Hilbert space in the following manner. Consider $E_{1}^{1}$, in the symmetric $|1 m\rangle$ basis, $m=1,0,-1$. The relationship between $|1 m\rangle$ basis and the computational basis is such that $|11\rangle=|\uparrow \uparrow\rangle,|10\rangle=\frac{|\uparrow \downarrow\rangle+|\downarrow \uparrow\rangle}{\sqrt{2}}$ and $|1-1\rangle=$ $|\downarrow \downarrow\rangle$. Here the spinors in the first and second positions correspond to the first and second qubits respectively. Let $U$ be the unitary matrix (orthogonal matrix, to be accurate, since Clebsch-Gordan coefficients are all real-valued) which transforms the computational basis to the angular momentum basis $|11\rangle,|10\rangle,|1-1\rangle,|00\rangle$. Then, the representation of $E_{1}^{1}$ in the 2-qubit state space of dimension 4 in the computational basis $|\uparrow \uparrow\rangle,|\uparrow \downarrow\rangle,|\downarrow \uparrow\rangle,|\downarrow \downarrow\rangle$, is given by

$$
\epsilon_{1}^{1}=U^{\dagger}\left(E_{1}^{1} \oplus 0\right) U, \quad \text { with } \quad U=\left(\begin{array}{cccc}
1 & 0 & 0 & 0 \\
0 & \frac{1}{\sqrt{2}} & \frac{1}{\sqrt{2}} & 0 \\
0 & 0 & 0 & 1 \\
0 & \frac{1}{\sqrt{2}} & -\frac{1}{\sqrt{2}} & 0
\end{array}\right),
$$

where $\oplus$ denotes the direct sum. Thus,

$$
\epsilon_{1}^{1}=\left(\begin{array}{cccc}
\frac{1}{6} & 0 & 0 & 0 \\
0 & \frac{1}{12} & \frac{1}{12} & 0 \\
0 & \frac{1}{12} & \frac{1}{12} & 0 \\
0 & 0 & 0 & 0
\end{array}\right), \quad \epsilon_{1}^{1}|\psi\rangle=\left(\begin{array}{cccc}
\frac{1}{6} & 0 & 0 & 0 \\
0 & \frac{1}{12} & \frac{1}{12} & 0 \\
0 & \frac{1}{12} & \frac{1}{12} & 0 \\
0 & 0 & 0 & 0
\end{array}\right)\left(\begin{array}{l}
a \\
b \\
c \\
d
\end{array}\right),
$$

and

$$
\frac{\epsilon_{1}^{1}|\psi\rangle}{\sqrt{\left\langle\psi\left|\epsilon_{1}^{1} \epsilon_{1}^{1}\right| \psi\right\rangle}}=\frac{\sqrt{2}}{\sqrt{\left(2 a^{2}+b^{2}+c^{2}+2 b c\right)}}\left(\begin{array}{c}
a \\
\frac{b+c}{2} \\
\frac{b+c}{2} \\
0
\end{array}\right),
$$

where $|\psi\rangle=a|\uparrow \uparrow\rangle+b|\uparrow \downarrow\rangle+c|\downarrow \uparrow\rangle+d|\downarrow \downarrow\rangle$, with $|a|^{2}+|b|^{2}+|c|^{2}+|d|^{2}=1$, is the most general pure state in the 2-qubit state space. Observe that the normalized resultant state is a symmetric state given by

$$
|\psi\rangle_{\text {sym }}=\frac{\sqrt{2} a|11\rangle}{\sqrt{\left(2 a^{2}+b^{2}+c^{2}+2 b c\right)}}+\frac{(b+c)|10\rangle}{\sqrt{\left(2 a^{2}+b^{2}+c^{2}+2 b c\right)}} .
$$

Therefore, $\epsilon_{1}^{1}$ projects a vector in the 4-dimensional Hilbert space onto the 3dimensional symmetric space.

These POVMs can be expressed in terms of Pauli spin matrices, which are identified as Hamiltonians easily implemented in NMR quantum computing. If $I_{1}$ and $I_{2}$ represent the identity matrices for the qubits 1 and 2 respectively, and $\sigma_{i}(1)$ and $\sigma_{i}(2)$, with $i=x, y, z$, the corresponding Pauli spin matrices, then $\epsilon_{1}^{1}$ 's can be expressed in terms of Pauli spin matrices as

$\epsilon_{1}^{1}=\frac{1}{6}\left[\frac{1}{2}\left(I_{1} \otimes I_{2}\right)+\frac{1}{4}\left(\sigma_{z}(1) \otimes I_{2}\right)+\frac{1}{4}\left(I_{1} \otimes \sigma_{z}(2)\right)+\frac{1}{4}\left(\sigma_{x}(1) \otimes \sigma_{x}(2)\right)+\frac{1}{4}\left(\sigma_{y}(1) \otimes \sigma_{y}(2)\right)\right]$, 
where the symbol $\otimes$ denotes the direct product. Similar calculations yield:

$$
\begin{aligned}
\epsilon_{0}^{0} & =\frac{1}{9}\left[\frac{3}{4}\left(I_{1} \otimes I_{2}\right)+\frac{1}{4}\left(\sigma_{z}(1) \otimes \sigma_{z}(2)\right)+\frac{1}{4}\left(\sigma_{y}(1) \otimes \sigma_{y}(2)\right)+\frac{1}{4}\left(\sigma_{x}(1) \otimes \sigma_{x}(2)\right)\right] ; \\
\epsilon_{0}^{1} & =\frac{1}{6}\left[\frac{1}{2}\left(I_{1} \otimes I_{2}\right)+\frac{1}{2}\left(\sigma_{z}(1) \otimes \sigma_{z}(2)\right)\right] ; \\
\epsilon_{-1}^{1} & =\frac{1}{6}\left[\frac{1}{2}\left(I_{1} \otimes I_{2}\right)-\frac{1}{4}\left(\sigma_{z}(1) \otimes I_{2}\right)-\frac{1}{4}\left(I_{1} \otimes \sigma_{z}(2)\right)+\frac{1}{4}\left(\sigma_{x}(1) \otimes \sigma_{x}(2)\right)+\frac{1}{4}\left(\sigma_{y}(1) \otimes \sigma_{y}(2)\right)\right] ; \\
\epsilon_{0}^{2} & =\frac{1}{12}\left[\left(I_{1} \otimes I_{2}\right)-\frac{1}{3}\left(\sigma_{z}(1) \otimes \sigma_{z}(2)\right) \quad+\frac{2}{3}\left(\sigma_{x}(1) \otimes \sigma_{x}(2)\right)+\frac{2}{3}\left(\sigma_{y}(1) \otimes \sigma_{y}(2)\right)\right] ; \\
\epsilon_{2}^{2} & =\frac{1}{12}\left[\left(I_{1} \otimes I_{2}\right)+\left(\sigma_{z}(1) \otimes I_{2}\right)+\left(I_{1} \otimes \sigma_{z}(2)\right)+\left(\sigma_{z}(1) \otimes \sigma_{z}(2)\right)\right] ; \\
\epsilon_{-2}^{2} & =\frac{1}{12}\left[\left(I_{1} \otimes I_{2}\right)-\left(\sigma_{z}(1) \otimes I_{2}\right)-\left(I_{1} \otimes \sigma_{z}(2)\right)+\left(\sigma_{z}(1) \otimes \sigma_{z}(2)\right)\right] .
\end{aligned}
$$

We have explicitly demonstrated the structure of $\epsilon_{q}^{k}$ for 2-qubits. In a similar way, it can be routinely calculated for 3-qubits and hence for n-qubits.

We turn our attention to the resulting state following a POVM measurement of a spin-1 density matrix . For a spin-1 system, the initial denssity matrix, in the representation given in (1), is given by

$$
\rho^{i}=\frac{1}{3}\left(\begin{array}{ccc}
1+\sqrt{\frac{3}{2}} t_{0}^{1}+\frac{1}{\sqrt{2}} t_{0}^{2} & \sqrt{\frac{3}{2}}\left(t_{-1}^{1}+t_{-1}^{2}\right) & \sqrt{3} t_{-2}^{2} \\
-\sqrt{\frac{3}{2}}\left(t_{1}^{1}+t_{1}^{2}\right) & 1-\sqrt{2} t_{0}^{2} & \sqrt{\frac{3}{2}}\left(t_{-1}^{1}-t_{-1}^{2}\right) \\
\sqrt{3} t_{2}^{2} & -\sqrt{\frac{3}{2}}\left(t_{1}^{1}-t_{1}^{2}\right) & 1-\sqrt{\frac{3}{2}} t_{0}^{1}+\frac{1}{\sqrt{2}} t_{0}^{2}
\end{array}\right):=\left(\begin{array}{c}
\rho_{11} \rho_{12} \rho_{13} \\
\rho_{21} \rho_{22} \rho_{23} \\
\rho_{31} \rho_{32} \rho_{33}
\end{array}\right) ;
$$

here $t_{q}^{k}$ with $k=1$ is the vector polarization parameter and $k=2$ is the tensor polarization parameter. From (3) we obtain,

$$
\rho_{10}^{f}=\frac{1}{\left(\rho_{11}+\rho_{33}\right)}\left(\begin{array}{ccc}
\rho_{11} & 0 & \rho_{13} \\
0 & 0 & 0 \\
\rho_{31} & 0 & \rho_{33}
\end{array}\right) .
$$

The only non-zero spherical tensor parameters are $t_{0}^{1}, t_{0}^{2}$ and $t_{ \pm 2}^{2}$. Such a system can be produced in the laboratory by the combined electric quadrupole and magnetic dipole field, where the direction of the magnetic field is along the $Z$-axis of the Principal Axis frame of the electric quadrupole field. In similar fashion, for $\rho_{11}^{f}$ and $\rho_{1-1}^{f}$, the non-zero spherical parameters are $t_{0}^{1}, t_{0}^{2}, t_{ \pm 1}^{1}$ and $t_{ \pm 1}^{2}$ whereas for $\rho_{20}^{f}$, all the spherical tensor parameters are non-zero. Also, we note that in the case of $\rho_{22}^{f}$ and $\rho_{2-2}^{f}$, an arbitrary initial state collapses to a separable state after performing a measurement with $E_{2}^{2}$ and $E_{-2}^{2}$.

Spin-j system- Since $\left(\tau_{q_{1}}^{k_{1}} \tau_{q_{2}}^{k_{2}}\right)=\sum_{k, q} C\left(k_{1} k_{2} k ; q_{1} q_{2} q\right) \tau_{q}^{k}$, for spin-j system where $j=n / 2$, our POVM becomes,

$$
E_{q^{\prime}}^{k^{\prime}}=(-1)^{q^{\prime}} \sum_{k} C\left(k^{\prime} k^{\prime} k ; q^{\prime}-q^{\prime} 0\right) \tau_{0}^{k}
$$

with $\tau_{0}^{k}(\mathbf{J})=\mathcal{N}_{k j}(\mathbf{J} \cdot \vec{\nabla})^{k} r^{k} Y_{0}^{k}(\hat{r})$ and $Y_{0}^{k}(\theta, \phi)=\sqrt{\frac{2 l+1}{4 \pi}} P_{l}(\cos \theta)$. Measurement 
on any POVM gives the linear combination of $\left\langle J_{z}^{k}\right\rangle$. Here $\left\langle J_{z}^{k}\right\rangle$ can be considered as the $k^{\text {th }}$ order moment of the statistical variate $J_{z}$, with $k=1, \ldots, 2 j$. If the given ensemble is a physical n-qubit system, the physical realization of POVM measurements can only be implemented by dilation, by going over to the $2^{n}$-dimensional Hilbert space.

Entangled states from separable ones. - It is well known that some of the projective measurements take separable states to entangled states. Considering a spin-1 example, again, we demonstrate how some of the constructed POVM measurements can be used to generate entangled states from separable states. Consider $\epsilon_{1}^{1}$ (constructed from $E_{1}^{1}$ ) acting on a separable state,

$$
|\psi\rangle=\frac{1}{2}\left(\begin{array}{l}
1 \\
1 \\
1 \\
1
\end{array}\right)=\left(\frac{|\uparrow(1)\rangle+|\downarrow(1)\rangle}{\sqrt{2}}\right) \otimes\left(\frac{|\uparrow(2)\rangle+|\downarrow(2)\rangle}{\sqrt{2}}\right) .
$$

Then, $\epsilon_{1}^{1}|\psi\rangle$ and the resulting normalized state $\left|\psi^{f}\right\rangle$ are

$$
\epsilon_{1}^{1}|\psi\rangle=\frac{1}{6}\left(\begin{array}{cccc}
1 & 0 & 0 & 0 \\
0 & \frac{1}{2} & \frac{1}{2} & 0 \\
0 & \frac{1}{2} & \frac{1}{2} & 0 \\
0 & 0 & 0 & 0
\end{array}\right)\left(\begin{array}{c}
\frac{1}{2} \\
\frac{1}{2} \\
\frac{1}{2} \\
\frac{1}{2}
\end{array}\right), \quad\left|\psi^{f}\right\rangle=\frac{\epsilon_{1}^{1}|\psi\rangle}{\sqrt{\left\langle\psi\left|\epsilon_{1}^{1} \epsilon_{1}^{1}\right| \psi\right\rangle}}=\frac{1}{\sqrt{3}}\left(\begin{array}{l}
1 \\
1 \\
1 \\
0
\end{array}\right) .
$$

In the computational basis, $\left|\psi^{f}\right\rangle$ can be represented as $\left|\psi^{f}\right\rangle=\frac{1}{\sqrt{3}}[|\uparrow \uparrow\rangle+|\uparrow \downarrow\rangle+|\downarrow \uparrow\rangle]$, with corresponding density matrix

$$
\rho_{11}^{f}=\left|\psi^{f}\right\rangle\left\langle\psi^{f}\right|=\frac{1}{3}\left(\begin{array}{llll}
1 & 1 & 1 & 0 \\
1 & 1 & 1 & 0 \\
1 & 1 & 1 & 0 \\
0 & 0 & 0 & 0
\end{array}\right) .
$$

In order to check if $\left|\psi^{f}\right\rangle$ is entangled, we use the PPT criterion, 32 which offers a necessary and sufficient condition for entanglement verification in $2 \times 2$ and $2 \times 3$ systems. The partially transposed matrix of $\rho_{11}^{f}$ in $(4)$ has eigenvalues $\lambda_{1}=0.872678, \lambda_{2}=-0.333333, \lambda_{3}=0.333333$, and $\lambda_{4}=0.127322$. Since $\lambda_{2}$ is negative, we conclude that a separable state collapses in to an entangled state after a measurement is performed with $\epsilon_{1}^{1}$.

\section{Conclusions}

We have exploited the vector space structure of the space of complex matrices in which the submanifold of density matrices resides to construct POVMs from any set of orthonormal basis matrices spanning the complex matrix space. The construction, using the notion of vectorisation, offers an interpretation of the POVMs as orthogonal projectors in a space of higher dimension. For the experimentally developed class of permutationally symmetric states particularly useful in quantum information processing, the POVMs constructed using the spherical tensor basis, are physically interpretable in terms of the angular momentum operators. Moreover, their physical realization as dilated measurements from the $n+1$-dimensional 
symmetric subspace to the $2^{n}$-dimensional Hilbert space using Clebsch-Gordan decomposition, presents a novel way to construct POVMs which always project onto space of symmetric states. This result is similar in spirit to Neumark's dilation of POVMs to projective measurements. For such measurements, repeatability can be interpreted in the sense that the result of a POVM acting on a state is always a permutationally symmetric state. Finally, we have shown that it is possible to generate entangled states from separable ones with the POVMs.

\section{Acknowledgements}

One of the authors (H.S.S) acknowledges with thanks the financial support provided by the Department of Science and Technology(DST), India for the award of INSPIRE fellowship.

\section{References}

1. F. Hirsch, M. T. Quintino, J. Bowles and N. Brunner Phys. Rev. Lett 111, (2013) 160402.

2. Ram A. Somaraju, Alain Sarlette and Hugo Thienpont, Proceeedings of 52nd IEEE Conference on Decision and Control, Florence, Italy (2013).

3. J. Wu, Int. J. Theor. Phys. 49 (2010) 324.

4. Z. Y. Wang,Int. J. Theor. Phys . 49 (2010)1357.

5. J. F. Song and Z. Y. Wang, Int. J. Theor. Phys. 50 (2011) 2410.

6. P. Zhou, J. Phys. A : Math. Theor. 45 (2012) 215305.

7. R. Blume-Kohout, J. O. S. Yin and S. J. van Enk, Phys. Rev. Lett 105 (2010) 170501.

8. S. Huang, Phys. Lett. A 377 (2013) 448 and the references therein.

9. T. Mor, arXiv:quant-th/9608005v1 (1996).

10. T. Mor and P. Horodecki, arXiv:quant-ph/9906039 (1999).

11. S. E. Ahnert and M. C. Payne, Phys. Rev. A 71 (2005) 012330.

12. M. Ziman and V. Buzek, Phys. Rev. A 72 (2005) 022343.

13. S. E. Ahnert and M. C. Payne, Phys. Rev. A 73 (2006) 022333.

14. C. M. Caves, C. A. Fuchs and R. Schack J. Math. Phys.43 (2002) 4537.

15. J. M. Rennes, R. Blume-Kohout, A. J. Scott and C. M. Caves J. Math. Phys. 45 (2004) 2171.

16. A. Kalev and G. Gour J. Phys. A: Math. Theor. 4 (2014)335302.

17. I. D. Ivanovic, Phys. Lett. A 123 (1987)257.

18. D. Dieks, Phys. Lett. A 126 (1988)303.

19. U. Fano Rev. Mod. Phys. 29 (1957) 74.

20. Blin- Stoyle, Grace.: Handbuck der Physik 62 Ed. SFlugge (Springer- Verlag, Berlin) (1957) 555.

21. G. Ramachandran, V. Ravishankar, S. N. Sandhya and Swarnamala Sirsi, J.Phys.G:Nucl.Phys., 13 (1987) L271-L273.

22. A. Peres, (Kluwer Academic Publishers, Dordecht, Netherlands, 1993).

23. H. Hlaffner, et al.: Nature 438 (2005) 643.

24. A. R. Usha Devi, Prabhu and R. Rajagopal, Phys. Rev. Lett. 98 (2007) 060501.

25. Z. Wang and D. Markham, Phys. Rev. Lett 108 (2012)210407.

26. C. Eltschka and J. Siewert, Phys. Rev. Lett 108 (2012) 230502.

27. C. Eltschka and J. Siewert, Phys. Rev. Lett 108 (2012)020502.

28. P. Ribeiro, and R. Mosseri, Phys. Rev. Lett 106 (2011)180502. 
12 Authors' Names

29. J. Preskill, Lecture notes for physics 229: quantum information and computation, 1998.

30. Satchler, et al.: Proceedings of the International Conference on Polarization Phenomena in Nuclear Reactions. University of Wisconsin Press, Madison (1971).

31. G. Racah, Group theory and spectroscopy. CERN, report 61-8 (1961).

32. A. Peres, Phys. Rev. Lett., 77 (1996) 1413. 